\title{
List of reviewers for 2010
}

The Journal of Neurodevelopmental Disorders would like to thank the following individuals for their reviews during 2010:

\author{
Brett Abrahams \\ Kristina Aldridge \\ Richard Anney \\ K Antshel \\ Carrie Bearden \\ Arthur Beaudet \\ Ayelet Ben Sasson \\ Nathalie Berube \\ Sven Bolte \\ Rita Cantor \\ Alice Carter \\ Carissa Cascio \\ Emrah Caylak \\ Lisa Cordeiro \\ Jacqueline Crawley \\ Stephen Dager \\ Diane Damiano \\ Peter de Jong \\ Etienne de Villers-Sidani \\ Darragh Devine \\ Manny DiCicco-Bloom \\ Joshua Diehl \\ Christine Ecker \\ Inge-Marie Eigsti \\ Susan Ellis Weismer
}

\author{
Simon Fisher \\ Susan Folstein \\ Joshua Gordon \\ Doron Gothelf \\ Daria Grafodatskaya \\ Brian Hallahan \\ Antonio Hardan \\ Susan Hepburn \\ Jane Herbert \\ David Hessl \\ Julie Hsu \\ Pingzhao $\mathrm{Hu}$ \\ Walter Kaufmann \\ Aaron Kemp \\ Bryan King \\ Bonnie Klein-Tasman \\ Ave Lachiewicz \\ Janine Lamb \\ Pat Levitt \\ Deborah Levy \\ Chantal Merette \\ Debbie Mills \\ Kazuhiko Nakabayashi \\ Marvin Natowicz \\ Robert Naviaux
}

\author{
Charles Nelson \\ Craig Newschaffer \\ Charles Njiokiktjen M.D. \\ Peggy Nopoulos \\ Courtenay Norbury \\ Erik O'Hanlon \\ Alistair Pagnamenta \\ Sarah Paterson \\ Liz Pellicano \\ David Picketts \\ Dalila Pinto \\ Daniela Plesa Skwerer \\ Isabelle Rapin \\ Armin Raznahan \\ Dene Robertson \\ Patricia Salinas \\ Curt Sandman \\ Richard Schwartz \\ Valerie Shafer \\ Lisa Strug \\ Therese van Amelsvoort \\ Jeremy Veenstra-VanderWeele \\ Laura Visu-Petra \\ Rick Wagner \\ Biran Yaspan
}

